PROCEEDINGS OF THE

AMERICAN MATHEMATICAL SOCIETY

Volume 130, Number 4 , Pages 1009-1017

S 0002-9939(01)06318-3

Article electronically published on August 29, 2001

\title{
THE UNCERTAINTY PRINCIPLE ON RIEMANNIAN SYMMETRIC SPACES OF THE NONCOMPACT TYPE
}

\author{
J. SENGUPTA
}

(Communicated by Rebecca Herb)

\begin{abstract}
The uncertainty principle in $\mathcal{R}^{n}$ says that it is impossible for a function and its Fourier transform to be simultaneously very rapidly decreasing. A quantitative assertion of this principle is Hardy's theorem. In this article we prove various generalisations of Hardy's theorem for Riemannian symmetric spaces of the noncompact type. In the case of the real line these results were obtained by Morgan and Cowling-Price.
\end{abstract}

\section{INTRODUCTION}

The uncertainty principle in harmonic analysis is the paradigm which says that it is impossible for a function and its Fourier transform to be simultaneously very rapidly decreasing. For instance in $\mathcal{R}^{n}$, the Paley-Wiener theorem tells us that the Fourier transform of an integrable function having compact support extends to an entire function on $\mathcal{C}^{n}$ and hence it cannot be compactly supported unless the function is zero almost everywhere. One of the very first results generalising this situation is Hardy's theorem whose statement is the following.

Theorem (Hardy 3, pp. 155-157], 8]). Let $f$ be a measurable function on $\mathcal{R}^{n}$ and denote by $\widehat{f}$ its Fourier transform. If $|f(x)| \leq A e^{-\alpha|x|^{2}}$ and $|\widehat{f}(\xi)| \leq B e^{-\beta|\xi|^{2}}$ where $A, B, \alpha, \beta$ are positive constants and $\alpha \beta>1 / 4$, then $f=0$ almost everywhere.

Hardy's theorem for $\mathcal{R}^{n}$ also includes a statement for the limiting case $\alpha \beta=1 / 4$. We do not discuss that situation here. Hardy's theorem can be generalised in various directions, e.g. we can have arbitrary conjugate exponent pairs $(p, q)$ and also in the $L^{p}-L^{q}$ context where $p$ and $q$ are not necessarily conjugate exponents. These generalisations are the strong version of Morgan's theorem and the Cowling-Price theorem for $\mathcal{R}^{n}$, respectively (cf. [2]).

Theorem (Morgan, strong version). Let $f: \mathcal{R}^{n} \rightarrow \mathcal{C}$ be measurable and assume that

(1) $|f(x)| \leq C e^{-\alpha|x|^{p}}$,

(2) $|\widehat{f}(\xi)| \leq C e^{-\beta|\xi|^{q}}$, where $C, \alpha, \beta$ are positive constants, $1<p<\infty$ and $1 / p+1 / q=1$.

If $(\alpha p)^{1 / p}(\beta q)^{1 / q}>1$, then $f=0$ almost everywhere.

Received by the editors September 18, 2000.

2000 Mathematics Subject Classification. Primary 43A85, 22E30.

(C)2001 American Mathematical Society 
Theorem (Cowling-Price). Let $f: \mathcal{R}^{n} \rightarrow \mathcal{C}$ be measurable and assume that

(1) $e^{\alpha|x|^{2}} f(x) \in L^{p}\left(\mathcal{R}^{n}\right)$,

(2) $e^{\beta|\xi|^{2}} \widehat{f}(\xi) \in L^{q}\left(\mathcal{R}^{n}\right)$, where $\alpha, \beta$ are positive constants and $1 \leq p, q \leq \infty$.

If $\alpha \beta>1 / 4$, then $f=0$ almost everywhere.

Remark 1.1. The two theorems above are not stated exactly in this form in [2]. However they may be derived rather easily from Beurling's theorem, namely Theorem 1.2 in [2. We omit the details.

The aim of this article is to prove the exact analogues of these two theorems for Riemannian symmetric spaces of the noncompact type. We will state our results in the next section where we will introduce the necessary notation.

\section{Notation And Statement of Results}

If $V$ is a finite dimensional real vector space, $V^{*}$ will denote its dual and $V_{\mathcal{C}}$ its complexification. If $\lambda \in V_{\mathcal{C}}^{*}$, then $\operatorname{Re} \lambda$ (resp. $\operatorname{Im} \lambda$ ) will denote the real (resp. imaginary) part of $\lambda$. Let $G$ be a connected noncompact semi-simple Lie group with finite centre, $K \subset G$ a maximal compact subgroup, $\theta$ the corresponding Cartan involution and $X=G / K$ the associated Riemannian symmetric space of the noncompact type. We will often identify (complex-valued) functions on $X$ with their pullbacks to $G$ without further comment. We recall that $G$ has Iwasawa decomposition $G=K A N$ and Cartan decomposition $G=K \mathrm{Cl}\left(A^{+}\right) K$ where $\mathrm{Cl}$ stands for closure. We denote the Lie algebra of $G$ by $\mathcal{G}$. Analogous notation will be used for Lie algebras of subgroups of $G$. The Killing form of $\mathcal{G}$ will be denoted by $\mathcal{B}$. The inner product on $\mathcal{A}$ as well as that on $\mathcal{A}^{*}$ induced by the Killing form will be indicated by $\langle$,$\rangle and the corresponding norm by ||$. We will denote by $M$ the centraliser of $A$ in $K$, and by $B=K / M$ the 'boundary' of the symmetric space $X$. Let $\Sigma(\mathcal{G}, \mathcal{A})$ be the set of restricted roots. $m_{\alpha}$ will be the multiplicity of the (restricted) root $\alpha$. We choose, once and for all, a set of positive restricted roots which we denote by $\Sigma^{+}$. The subset of positive indivisible restricted roots will be denoted by $\Sigma_{0}^{+}$. As usual, $\rho$ will stand for the half-sum of the positive restricted roots, counted with multiplicities. The symbol $W$ will be used for the (little) Weyl group.

Let $o=e K$ denote the 'origin' in $X$ and let $d$ be the distance function on $X$ induced by the Riemannian metric of $X$. Let $\sigma: G \rightarrow \mathcal{R}$ be the function $\sigma(g)=d(o, g K)$. The function $\sigma$ is nonnegative, continuous, $K$-bi-invariant and $\sigma(g)=\sigma\left(g^{-1}\right)$ for all $g \in G$. Furthermore, $\sigma(\exp H)=|H|=\mathcal{B}(H, H)^{1 / 2}$ for all $H \in \mathcal{A}$.

We denote by $d x$ the $G$-invariant measure on $X$ and by $d k$ the normalised Haar measure of $K$. Let $d b$ be the $K$-invariant probability measure on $B$. We denote by $d a($ resp. $d n)$ the Haar measure on $A$ (resp. $N$ ). Finally $d \lambda$ will denote Lebesgue measure on $\mathcal{A}^{*}$. These are all normalised as in [5, pp. 100-101] to which we refer for further explanation.

We will employ Helgason's definition of the Fourier transform on $X$ ([5], Chapter 3 ), which is the appropriate Fourier transform in this context. Let $H: G \rightarrow \mathcal{A}$ be the Iwasawa projection and let $A: X \times B \rightarrow \mathcal{A}$ be given by $A(x, b)=-H\left(g^{-1} k\right)$ for $x=g K \in X$ and $b=k M \in B$. Then $A$ is real analytic. We recall that if $f$ is a 
(complex-valued) function on $X$, the Fourier transform $\tilde{f}$ is defined by

$$
\tilde{f}(\lambda, b)=\int_{X} f(x) e^{(-i \lambda+\rho)(A(x, b))} d x
$$

for all $\lambda \in \mathcal{A}_{\mathcal{C}}^{*}$ and $b \in B$ for which this integral converges.

We will also need the Radon transform on $X$ and its relation with the Fourier transform. The Radon transform of a function $f$ on $X$ will be denoted by $R f$. It is defined by

$$
R f(b, a)=R f(k M, a)=e^{\rho(\log a)} \int_{N} f(k a n \cdot o) d n .
$$

If $f \in L^{1}(X)$, then $R f \in L^{1}(B \times A ; d b d a)$ and $f \rightarrow R f$ is a continuous linear map of $L^{1}(X)$ into $L^{1}(B \times A)$. This can be easily seen by considering the function

$$
f^{\sharp}(x)=\int_{K} f(k \cdot x) d x .
$$

Now $f^{\sharp}$ is $K$-invariant and the Abel transform $\mathbf{A}: L^{1}(K \backslash G / K) \longrightarrow L^{1}(A)^{W}$ is a continuous linear map. Furthermore, $R$ is injective ([5, p. 104]).

Remark 2.1. Our definition of the Radon transform differs from that in [5] by the factor $e^{\rho(\log a)}$. Our definition is well adapted to our needs and has been previously employed in [1]. In particular if $f \in L^{1}(X)$ is $K$-invariant, then $R f$ coincides with the Abel transform $\mathbf{A} f$ of $f$.

Perhaps the most important property of the Radon transform is the following ([5. p. 276]). For suitably decaying functions $f$ on $X$, the Fourier transform $\tilde{f}$ is the $A$-Euclidean Fourier transform of its Radon transform $R f$, i.e.,

$$
\tilde{f}(\lambda, b)=\mathcal{F}(R f)(\lambda, b)=\int_{A} R f(b, a) e^{-i \lambda(\log a)} d a .
$$

Here $\mathcal{F}$ denotes the Euclidean Fourier transform on $A$.

We will utilise the above connection between the Fourier transform and the Radon transform repeatedly. We are now in a position to state our results.

Theorem 1. Let $f: X \rightarrow \mathcal{C}$ be measurable and assume that for all $x \in X, \lambda \in \mathcal{A}^{*}$ and $b \in B$ we have

(1) $|f(x)| \leq C e^{-\alpha \sigma^{p}(x)}$,

(2) $|\tilde{f}(\lambda, b)| \leq C e^{-\beta|\lambda|^{q}}$, where $C, \alpha, \beta$ are positive constants, $1<p<\infty$ and $1 / p+1 / q=1$.

$$
\text { If }(\alpha p)^{1 / p}(\beta q)^{1 / q}>1 \text {, then } f=0 \text { almost everywhere. }
$$

Theorem 2. Let $f: X \rightarrow \mathcal{C}$ be measurable and assume that for positive constants $\alpha$ and $\beta$ we have

(1) $e_{\alpha} f \in L^{p}(X)$,

(2) $e_{\beta} \tilde{f} \in L^{q}\left(\mathcal{A}^{*} \times B ;|c(\lambda)|^{-2} d \lambda d b\right)$, where $1 \leq p, q \leq \infty, \quad e_{\alpha}(x)=e^{\alpha \sigma^{2}(x)}$ and $e_{\beta}(\lambda)=e^{\beta|\lambda|^{2}}$.

If $\alpha \beta>1 / 4$, then $f=0$ almost everywhere. 


\section{Proof of the theorems}

As mentioned earlier we will reduce the theorems to the Euclidean situation via the Radon transform. We will first prove the following proposition.

Proposition 1. Let $f$ be as in Theorem 11. For each $\alpha^{1}$ with $0<\alpha^{1}<\alpha$, there exists a constant $E>0$ such that $|R f(b, a)| \leq E e^{-\alpha^{1} \sigma^{p}(a)}$ for all $(b, a) \in B \times A$.

Proof. By the definition of $R f$ we have

$$
\begin{aligned}
|R f(b, a)| & \leq e^{\rho(\log a)} \int_{N} \mid f(\text { kan.o }) \mid d n \\
& \leq C e^{\rho(\log a)} \int_{N} e^{-\alpha \sigma^{p}(a n)} d n \quad \text { (since } \sigma \text { is } K \text {-invariant) } \\
& =C e^{\rho(\log a)} \int_{N} \Xi(\text { an }) e^{-\alpha \sigma^{p}(a n)} \Xi(\text { an })^{-1} d n,
\end{aligned}
$$

where $\Xi$ is the zonal spherical function with parameter 0 .

Let $I$ denote the integral occurring in the last line of the previous formula. Recall that $\sigma(a n) \geq \sigma(a)$ for all $a \in A$ and $n \in N$. Hence for any $0<\alpha^{1}<\alpha$, the integral above is $\leq e^{-\alpha^{1} \sigma^{p}(a)} \int_{N} \Xi(a n) e^{-\epsilon \sigma^{p}(a n)} \Xi(a n)^{-1} d n$, where $\epsilon=\alpha-\alpha^{1}$. Now $\Xi(g) \geq e^{-|\rho| \sigma(g)}$ for all $g \in G$, and therefore $\Xi(a n)^{-1} \leq e^{|\rho| \sigma(a n)}$. Hence

$$
\begin{aligned}
I & \leq \int_{N} \Xi(\text { an }) e^{-\epsilon \sigma^{p}(a n)+|\rho| \sigma(a n)} d n \\
& =\int_{N} \Xi(\text { an }) e^{-\epsilon \sigma^{p}(a n)+|\rho| \sigma(a n)}(1+\sigma(\text { an }))^{-l_{1}}(1+\sigma(\text { an }))^{l_{1}} d n,
\end{aligned}
$$

where $l_{1}$ is any positive integer.

Now

$$
D:=\sup _{g \in G} e^{-\epsilon \sigma^{p}(g)+|\rho| \sigma(g)}(1+\sigma(g))^{l_{1}}<\infty
$$

Therefore

$$
I \leq D \int_{N} \Xi(a n)(1+\sigma(a n))^{-l_{1}} d n .
$$

Now given any nonnegative integer $m$, there is a positive integer $m_{1}$ such that for all $a \in A$ we have the estimate (4, p. 264)

$$
e^{\rho(\log a)} \int_{N} \Xi(a n)(1+\sigma(a n))^{-m_{1}} d n \leq \tilde{C}(1+\sigma(a))^{-m},
$$

where $\tilde{C}>0$ is a constant. Choosing $m=0$ and $l_{1}=m_{1}$ immediately yields our proposition.

Proof of Theorem [1. We have $\tilde{f}=\mathcal{F}(R f)$. Choose $0<\alpha^{1}<\alpha$ such that

$$
\left(\alpha^{1} p\right)^{1 / p}(\beta q)^{1 / q}>1
$$

Then Morgan's theorem for the vector group $A$ says that for all $b \in B$, the function $a \rightarrow R f(b, a)$ is equal to 0 almost everywhere on $A$. This implies that for all $b \in B, \tilde{f}(\lambda, b)=0 \forall \lambda \in \mathcal{A}^{*}$, which in turn implies that the $L^{2}$ norm of $f$ is zero by the Plancherel theorem for $X$. Hence $f=0$ almost everywhere. 
Remark 3.1. In the case $p=q=2$, Theorem 1 provides a proof of Hardy's theorem, which is different to the one given in [7].

The proof of Theorem 2 is similar in spirit to that of Theorem 1 but is technically more involved. We first note that the case $p=q=\infty$ is contained in the previous theorem. Hence we can assume that at least one of $p$ and $q$ is finite. We will first assume that $p$ and $q$ are both finite. We begin with the following observation. Let $f$ be as in Theorem 2 and let $\psi \in \mathcal{D}(K \backslash G / K)$, the space of smooth, compactly supported, $K$-bi-invariant functions on $G$. Let $h=f * \psi$ where $*$ denotes convolution. Note that $h$ is a smooth function on $G$ which is right $K$-invariant. We will first prove the following lemma.

Lemma 2. $e_{\alpha^{1}} h \in L^{1}(G)$ for any $\alpha^{1}$ with $0 \leq \alpha^{1}<\alpha$.

Proof. Let

$$
I=\int_{G} e^{\alpha^{1} \sigma^{2}(z)}|h(z)| d z
$$

We have to show that $I$ is finite. Now $|h(z)| \leq \int_{G}|f(y)|\left|\psi\left(y^{-1} z\right)\right| d y$.

Applying Fubini's theorem we therefore obtain

$$
\begin{aligned}
I & \leq \int_{G} e^{\alpha^{1} \sigma^{2}(z)}\left(\int_{G}|f(y)|\left|\psi\left(y^{-1} z\right)\right| d y\right) d z \\
& =\int_{G} \int_{G} e^{\alpha^{1} \sigma^{2}(z)}\left|\psi\left(y^{-1} z\right)\right||f(y)| d z d y .
\end{aligned}
$$

We look at

$$
I_{1}(y):=\int_{G} e^{\alpha^{1} \sigma^{2}(z)}\left|\psi\left(y^{-1} z\right)\right| d z .
$$

Making the change of variable $x=y^{-1} z$, we obtain that

$$
I_{1}(y)=\int_{G} e^{\alpha^{1} \sigma^{2}(y x)}|\psi(x)| d x .
$$

As a function of $y \in G, I_{1}$ is $K$-bi-invariant. Thus it is completely determined by its restriction to $A$. So let $y=a \in A$. Then we obtain

$$
I_{1}(a) \leq e^{\alpha^{1}(\sigma(a)+M)^{2}} \int_{G}|\psi(x)| d x
$$

where $M=\sup (\sigma(x) \mid x \in$ support of $\psi)$.

We now choose $\tilde{\alpha}$ such that $\alpha^{1}<\tilde{\alpha}<\alpha$. Then we know that $e_{\tilde{\alpha}} f \in L^{1}(G)$. Hence letting $|\psi|$ denote the $L^{1}$ norm of $\psi$, we get

$$
\begin{aligned}
I & \leq|\psi| \int_{G} e^{\alpha^{1}(\sigma(y)+M)^{2}}|f(y)| d y \\
& =|\psi| \int_{G} e_{\tilde{\alpha}}(y)|f(y)| e^{\alpha^{1}(\sigma(y)+M)^{2}-\tilde{\alpha} \sigma^{2}(y)} d y .
\end{aligned}
$$

This proves $I<\infty$ because

$$
\sup _{y \in G} e^{\alpha^{1}(\sigma(y)+M)^{2}-\tilde{\alpha} \sigma^{2}(y)}<\infty .
$$


We recall that the Fourier transform of $f, \tilde{f}(\lambda, b)$, is defined by

$$
\tilde{f}(\lambda, b)=\int_{X} e^{(-i \lambda+\rho)(A(x, b))} f(x) d x .
$$

Condition (1) in Theorem 2 implies that $e_{\alpha^{1}} f \in L^{1}(X)$ for all $\alpha^{1}$ with $0<\alpha^{1}<$ $\alpha$. We choose such an $\alpha^{1}$ and keep it fixed. We have $|A(x, b)| \leq F \sigma(x)$ for all $x \in X$ and $b \in B$ where $F$ is a positive constant (see 4, p. 167]). These facts imply that the integral defining $\tilde{f}(\lambda, b)$ is absolutely convergent for all $\lambda \in \mathcal{A}_{\mathcal{C}}^{*}$ and $b \in B$. We will sketch a proof of this below.

We have for all $\lambda \in \mathcal{A}_{\mathcal{C}}^{*}$ and $b \in B$,

$$
\begin{aligned}
|\tilde{f}(\lambda, b)| & \leq \int_{X}\left|e^{(-i \lambda+\rho)(A(x, b))} f(x)\right| d x \\
& \leq \int_{X} e^{|\operatorname{Im} \lambda+\rho| F \sigma(x)}|f(x)| d x \\
& =\int_{X} e^{|\operatorname{Im} \lambda+\rho| F \sigma(x)-\alpha^{1} \sigma^{2}(x)} e_{\alpha_{1}}(x)|f(x)| d x
\end{aligned}
$$

Now

$$
D(\operatorname{Im} \lambda):=\sup _{x \in X} e^{|\operatorname{Im} \lambda+\rho| F \sigma(x)-\alpha^{1} \sigma^{2}(x)}<\infty .
$$

Hence $|\tilde{f}(\lambda, b)| \leq D(\operatorname{Im} \lambda) \int_{X} e_{\alpha^{1}}(x)|f(x)| d x<\infty$ and we are done. This argument also shows that $\tilde{f}(\lambda, b)$ is a bounded function on $\mathcal{A}^{*} \times B$.

Lebesgue's dominated convergence theorem shows that $\tilde{f}(\lambda, b)$ is a continuous function on $\mathcal{A}^{*} \times B$. Furthermore, Morera's theorem in conjunction with Fubini's theorem shows that for fixed $b \in B$, the function $\mathcal{A}_{\mathcal{C}}^{*} \ni \lambda \rightarrow \tilde{f}(\lambda, b)$ is a holomorphic function on $\mathcal{A}_{\mathcal{C}}^{*}$. In particular, for fixed $b \in B$, the function $\mathcal{A}^{*} \ni \lambda \rightarrow \tilde{f}(\lambda, b)$ is a real analytic function on $\mathcal{A}^{*}$. Furthermore, since $\tilde{f}(\lambda, b)$ is a bounded function on $\mathcal{A}^{*} \times B$ the Plancherel theorem for $X$ implies that $f \in L^{2}(X)$ because of condition (2) in Theorem 2 .

Proof of Theorem 2, It follows from the assumptions that

$$
e_{\beta^{1}} \tilde{f} \in L^{1}\left(\mathcal{A}^{*} \times B ;|c(\lambda)|^{-2} d \lambda d b\right)
$$

for all $\beta^{1}$ with $0<\beta^{1}<\beta$.

As in the proof of Theorem 1, we will show that for almost every $b \in B$, the function $a \rightarrow R f(b, a)$ has the property $e_{\alpha^{1}}(a) R f(b, a) \in L^{1}(A ; d a)$ where $\alpha^{1}$ is as in Lemma 2. We choose $\tilde{\alpha}$ such that $\alpha^{1}<\tilde{\alpha}<\alpha$ and set $g(y)=e_{\tilde{\alpha}}(y)|h(y)|$ for $y \in G$. Then $g$ is a continuous right- $K$-invariant function on $G$ which is integrable. Hence, since $\sigma$ is $K$-invariant,

$$
\begin{aligned}
\infty & >\int_{K} \int_{A} \int_{N} g(k a n) e^{2 \rho(\log a)} d k d a d n \\
& =\int_{K} \int_{A} \int_{N} e^{\tilde{\alpha} \sigma^{2}(k a n)+2 \rho(\log a)}|h(k a n)| d k d a d n \\
& =\int_{K} \int_{A} \int_{N} e^{\tilde{\alpha} \sigma^{2}(a n)+2 \rho(\log a)}|h(k a n)| d k d a d n
\end{aligned}
$$


Now $\sigma($ an $) \geq \sigma(a)$ for all $a \in A$ and $n \in N$. Hence

$$
\int_{K} \int_{A} \int_{N} e^{\tilde{\alpha} \sigma^{2}(a)+2 \rho(\log a)}|h(k a n)| d k d a d n<\infty .
$$

Fubini's theorem therefore implies that for almost every $(k, a) \in K \times A$

$$
\int_{N} e^{\tilde{\alpha} \sigma^{2}(a)+2 \rho(\log a)}|h(k a n)| d n<\infty,
$$

i.e., $e^{\tilde{\alpha} \sigma^{2}(a)+\rho(\log a)} R(|h|)(k, a)<\infty$ for almost every $(k, a)$, and

$$
\int_{K} \int_{A} e^{\tilde{\alpha} \sigma^{2}(a)+\rho(\log a)} R(|h|)(k, a) d k d a<\infty .
$$

We know that $e^{\rho(\log a)} \leq e^{|\rho| \sigma(a)}$. Let $\epsilon=\tilde{\alpha}-\alpha^{1}$. Then $\epsilon>0$ and

$$
\begin{aligned}
\int_{K} \int_{A} e^{\alpha^{1} \sigma^{2}(a)}|R h(k, a)| d k d a \leq \int_{K} \int_{A} e^{\alpha^{1} \sigma^{2}(a)} R(|h|)(k, a) d k d a \\
\quad=\int_{K} \int_{A} e^{\tilde{\alpha} \sigma^{2}(a)+\rho(\log a)} e^{-\epsilon \sigma^{2}(a)-\rho(\log a)} R(|h|)(k, a) d k d a \\
\quad<\infty
\end{aligned}
$$

because $|R h(k, a)| \leq R(|h|)(k, a)$ and

$$
\sup _{a \in A} e^{-\epsilon \sigma^{2}(a)-\rho(\log a)} \leq \sup _{a \in A} e^{-\epsilon \sigma^{2}(a)+|\rho| \sigma(a)}<\infty .
$$

From Fubini's theorem we conclude that for almost every $b \in B$, the function $a \rightarrow e^{\alpha^{1} \sigma^{2}(a)} R h(b, a) \in L^{1}(A ; d a)$.

We have the following estimate for the Plancherel density $|c(\lambda)|^{-2}$ (see [1, p. 394]):

$$
|c(\lambda)|^{-2} \asymp \prod_{\alpha \in \Sigma_{0}^{+}}\langle\lambda, \alpha\rangle^{2}\left(1+|\langle\lambda, \alpha\rangle|^{2}\right)^{m_{\alpha}+m_{2 \alpha}-2} \quad\left(\lambda \in \mathcal{A}^{*}\right) .
$$

This shows that for any $\beta^{1}, 0 \leq \beta^{1}<\beta$, we have

$$
e_{\beta^{1}} \tilde{f} \in L^{1}\left(\mathcal{A}^{*} \times B ; \prod_{\alpha \in \Sigma_{0}^{+}}\langle\lambda, \alpha\rangle^{2} d \lambda d b\right) .
$$

Let $D$ be the constant coefficient differential operator on $A \cong \mathcal{A}$ whose symbol is $\prod_{\alpha \in \Sigma_{0}^{+}}\langle\lambda, \alpha\rangle^{2}$, i.e., $D$ corresponds to multiplication by the $W$-invariant polynomial $\prod_{\alpha \in \Sigma_{0}^{+}}\langle\lambda, \alpha\rangle^{2}$ under $\mathcal{F} . D$ is $W$-invariant. We will denote the ring of $G$-invariant differential operators on $X$ (resp. the ring of $W$-invariant constant coefficient differential operators on $A$ ) by $D(X)$ (resp. $\left.D_{W}(A)\right)$. There is a surjective isomorphism $\Gamma: D(X) \rightarrow D_{W}(A)$ (see [5, p. 87]). Let $\tilde{D}$ be the unique element of $D(X)$ such that $\Gamma(\tilde{D})=D$. Now $\tilde{D} h=f * \tilde{D} \psi$ and $\tilde{D} \psi \in \mathcal{D}(K \backslash G / K)$. In view of our earlier results we know that the function $a \rightarrow R \tilde{D} h(b, a) \in L^{1}(A ; d a)$ for almost every $b \in B$. Furthermore,

$$
\begin{aligned}
\mathcal{F}(R \tilde{D} h(b, a)) & =(\tilde{D} h)^{\sim}(\lambda, b) \\
& =(f * \tilde{D} \psi)^{\sim}(\lambda, b) \\
& =\tilde{f}(\lambda, b)(\tilde{D} \psi)^{\sim}(\lambda) \\
& =\tilde{f}(\lambda, b) \prod_{\alpha \in \Sigma_{0}^{+}}\langle\lambda, \alpha\rangle^{2} \tilde{\psi}(\lambda)
\end{aligned}
$$


since $\tilde{D} \psi$ is $K$-invariant (see Lemma 1.4 in Chapter 3 of [5] and its proof and Theorem 6.2.2 [5 p. 262]). Now $\tilde{\psi}$ is a bounded real analytic function on $\mathcal{A}^{*}$. Hence $e_{\beta^{1}}(\tilde{D} h)^{\sim} \in L^{1}\left(\mathcal{A}^{*} \times B ; d \lambda d b\right) \forall \beta^{1}, 0 \leq \beta^{1}<\beta$.

Now given $\alpha>0, \beta>0$ with the property $\alpha \beta>1 / 4$ we can find $\alpha^{1}>0, \beta^{1}>0$ such that $\alpha^{1}<\alpha, \beta^{1}<\beta$ and $\alpha^{1} \beta^{1}>1 / 4$. The Euclidean Cowling-Price theorem for the vector group $A$ applied to the Fourier transform pair $R \tilde{D} h(., a), \mathcal{F} R \tilde{D} h(., a)$ tells us that for almost every $b \in B$ the function $a \rightarrow R \tilde{D} h(b, a)$ is zero almost everywhere on $A$. This implies that for almost every $b \in B$, the real analytic function $\tilde{f}(\lambda, b) \prod_{\alpha \in \Sigma_{0}^{+}}\langle\lambda, \alpha\rangle^{2} \tilde{\psi}(\lambda)$ vanishes identically on $\mathcal{A}^{*}$. Now the real analytic functions $\tilde{\psi}$ and $\prod_{\alpha \in \Sigma_{0}^{+}}\langle\lambda, \alpha\rangle^{2}$ do not vanish identically on $\mathcal{A}^{*}$ (since $\psi \neq$ $0)$. We therefore conclude that $\tilde{f}(\lambda, b)=0 \forall \lambda \in \mathcal{A}^{*}$ for almost every $b \in B$. Since $f \in L^{2}(X)$, the Plancherel theorem for $X$ tells us that the $L^{2}$ norm of $f$ is zero. Hence $f=0$ almost everywhere, as desired.

An inspection of the proof above shows that the same arguments remain valid when one of $p$ or $q$ is infinite, in fact, they become slightly simpler. This concludes the proof of Theorem 2 .

Remark 3.2. In certain special cases, e.g. when $f$ is $K$-invariant and the group $G$ has real rank one or has only one conjugacy class of Cartan subgroups, Theorem 2 can be deduced from Theorem 1] This can be seen by using the method of Bagchi-Ray described in [2] and invoking our Proposition 1. We note that if $G$ is as above the Plancherel density $|c(\lambda)|^{-2}$ has the property $|c(\lambda)|^{-2} \gg P(\lambda)$ where $P(\lambda)$ is a $W$-invariant polynomial. The polynomial $P$ corresponds to a $G$-invariant differential operator on $X$. In the general higher rank case, the Plancherel density does not have the above mentioned property and the method pursued in [2] fails since we cannot manufacture the appropriate $G$-invariant differential operator on $X$ from the Plancherel density. It appears very likely therefore that, in general, Theorem 2 cannot be deduced from Theorem 1

Remark 3.3. The referee has kindly informed the author that Narayanan and Ray (see [6]) have proved the analogue of the Cowling-Price theorem for the full group $G$. Their approach is different from ours.

\section{ACKNowledgement}

The author would like to thank the referee for suggestions which helped to improve the exposition.

\section{REFERENCES}

[1] J.P. Anker, A basic inequality for scattering theory on Riemannian symmetric spaces of the noncompact type, Am. J. Math. 113 (1991), 391-398. MR 92k:43008

[2] S.C. Bagchi and S.K. Ray, Uncertainty principles like Hardy's theorem on some Lie groups, J. Aust. Math. Soc. Series A 65 (1998), 289-302. MR 99k:43001

[3] H. Dym and H.P. McKean, Fourier series and integrals, Academic Press, New York, 1972. MR 56:945

[4] R. Gangolli and V.S. Varadarajan, Harmonic analysis of spherical functions on real reductive Lie groups, Springer-Verlag, 1988. MR 89m:22015

[5] S. Helgason, Geometric analysis on symmetric spaces, Mathematical Surveys and Monographs vol 39 AMS, 1994. MR 96h:43009

[6] Narayanan and Ray, $L^{p}$ versions of Hardy's theorem on semi-simple groups, Proc. AMS to appear. 
[7] A. Sitaram and M. Sundari, An analogue of Hardy's theorem for very rapidly decreasing functions on semi-simple Lie groups, Pacific J. Math. 177 (1997), 187-200. MR 99a:22018

[8] A. Sitaram, M.Sundari and S.Thangavelu, Uncertainty principles on certain Lie groups, Proc. Ind. Acad. Sci. (Math. Sci.) 105 (1995), 133-151. MR 96h:43002

School of Mathematics, Tata Institute of Fundamental Research, Homi Bhabha ROAD, BOMBAY - 400 005, INDIA

E-mail address: sengupta@math.tifr.res.in 